Article

\title{
Understanding Separation Mechanisms of Monoatomic Gases, Such as Kr and Xe, via DD3R Zeolite Membrane Using Molecular Dynamics
}

\author{
Bandar J. Bashmmakh ${ }^{1}\left(\mathbb{D}\right.$, Xiaoyu Wang ${ }^{2} \mathbb{D}$, Cynthia J. Jameson $^{3} \mathbb{D}$ and Sohail Murad ${ }^{1, *(\mathbb{D})}$ \\ 1 Department of Chemical and Biological Engineering, Illinois Institute of Technology, Chicago, IL 60616, USA; \\ bbashmmakh@hawk.iit.edu \\ 2 Department of Chemical and Biomolecular Engineering, University of Notre Dame, Notre Dame, IN 46556, USA; \\ xwang58@nd.edu \\ 3 Department of Chemistry, University of Illinois Chicago, Chicago, IL 60607, USA; cjjames@uic.edu \\ * Correspondence: murad@iit.edu
}

check for

updates

Citation: Bashmmakh, B.J.; Wang, X.;

Jameson, C.J.; Murad, S.

Understanding Separation

Mechanisms of Monoatomic Gases,

Such as $\mathrm{Kr}$ and $\mathrm{Xe}$, via DD3R Zeolite

Membrane Using Molecular

Dynamics. Thermo 2022, 2, 56-73.

https://doi.org/10.3390/

thermo2010005

Academic Editor: Johan Jacquemin

Received: 14 January 2022

Accepted: 21 February 2022

Published: 23 February 2022

Publisher's Note: MDPI stays neutral with regard to jurisdictional claims in published maps and institutional affiliations.

Copyright: (C) 2022 by the authors. Licensee MDPI, Basel, Switzerland. This article is an open access article distributed under the terms and conditions of the Creative Commons Attribution (CC BY) license (https:// creativecommons.org/licenses/by/ $4.0 /)$.

\begin{abstract}
Noble gas fission byproducts, such as $\mathrm{Kr}$ and $\mathrm{Xe}$, are generated within nuclear power reactors are currently being discharged into the atmosphere. This practice has a major economic drawback because of the high value associated with some of these gases. The separations of these gases are economically prohibitive because of the high energy requirement associated with cryogenic distillation. Zeolites, nanoporous materials suitable for gas separation processes, have exhibited high selectivity for such separations. We have used nonequilibrium molecular dynamics (MD) to investigate the separation performance of DD3R framework zeolitic membrane. The effects of pressure, temperature, and pure vs. mixture gas feed conditions are studied in this work to understand and explain, at the molecular level, the mechanisms of these $(\mathrm{Kr} / \mathrm{Xe})$ separations. Our studies have shown that the DD3R membrane shows promise for high selectivity ratios of Kr over Xe. MD runs show agreement with experimental trends of the permeation of $\mathrm{Kr} / \mathrm{Xe}$ pure and mixed gases using DD3R zeolite with high separation factor. Despite the absence of Xe complete permeation through the membrane because of MD timescale limitations, our results are sufficient to describe the mechanisms of these separations.
\end{abstract}

Keywords: gas permeability; zeolite; membrane-based separation; molecular dynamics simulations

\section{Introduction}

Nuclear energy, despite being unfavored for a long time, is being seriously reconsidered as a clean, reliable alternative energy source due to its minimal contribution to global warming in comparison to other energy sources such as fossil-fuels. This is a big motivation for research that aims to make nuclear power a safer and economically feasible energy alternative, even when compared to intermittent renewable energy sources. For this to be achieved, some practices in nuclear power generation require some change.

Currently, noble gases, such as radioactive ${ }^{85} \mathrm{Kr}$ isotope and sTable $1^{35} \mathrm{Xe}$, generated as fission byproducts in nuclear power reactors, are being discharged into the atmosphere. Although radiation level increases are limited, radioactive gases should be stored until stable. Unfortunately, the volume occupied by this gas is prohibitively large and represent a major challenge to store. Meanwhile, almost three quarters of the volume of this gas is sTable $1^{35} \mathrm{Xe}$, which provides an economical motive due to its high market value.

For a long time, $\mathrm{Kr} / \mathrm{Xe}$ separations were done using cryogenic distillation, which is associated with high energy requirement. Adsorption on nanoporous materials, a process shown to be a good alternative to cryogenic distillation, has two important characteristics: the high surface area that allows for higher gas uptake, caused by the unique-shaped cages within zeolite crystalline structures, and the potential enhancement of selectivity 
by chemical fine tuning to acquire desired adsorbate-adsorbent interactions. Zeolitic molecular sieves are categorized among the industrial nanoporous materials most used for gas separation. Based on characteristics including pore size, cage occupancy numbers, and void fraction of different zeolite frameworks, a wide range of values for $\mathrm{Kr} / \mathrm{Xe}$ selectivity factors are found; the adsorption selectivity of zeolites is favorable toward Xe.

For example, Linde Type A (NaA) zeolite has shown $\mathrm{Xe} / \mathrm{Kr}$ adsorption selectivity factors as high as 4.6 at $1 \mathrm{~atm}$ and $300 \mathrm{~K}$ for an equimolar mixture [1]. NaA zeolite has a uniform aperture size of 4.1 , which is larger than both kinetic diameters $(\mathrm{d}(\mathrm{Kr})=3.69 \AA$ and $\mathrm{d}(\mathrm{Xe})=4.05 \AA$ ) making diffusion selectivity in favor for $\mathrm{Xe}$, an unfavorable outcome. Chabazite (CHA) zeolite, on the other hand, has an aperture size of $3.8 \AA$ resulting in barrier-height-limited permeation conditions in favor of $\mathrm{Kr}$ with $\mathrm{Kr} / \mathrm{Xe}$ separation factor as high as 51 [2].

Experimental work for such separations has been carried out for deca-dodecasilrhombohedral (DD3R) zeolite frameworks, with promising $\mathrm{Kr} / \mathrm{Xe}$ separation factors [3]. In the experiments, DD3R zeolite membranes fabricated on four-channel hollow fibers had a thickness of $\sim 4.4 \mu \mathrm{m}$, and were highly robust and defect-free. The single gas permeation and mixed gas separation performance were evaluated by the Wicke-Kallenbach technique. The experimental details are described in detail in an earlier work and its supporting information [4]. The earlier gas separation experiments were carried out using the same Wicke-Kallenbach technique described in [5]. The partial pressures in the permeate and retentate side were measured with a Ledamass Quadrupole Mass Analyzer. Although the commercial sources for the DD3R crystals and the membranes were listed in the acknowledgment, no description was provided [6].

According to experiments, $\mathrm{Kr}$ permeation through DD3R membranes is higher in the mixture case in comparison to the individual pure gases. In addition, $\mathrm{Kr}$ pure gas permeance is higher than that of pure Xe [3].

In the present work, we used non-equilibrium molecular dynamics (MD), as implemented by [4] for $\mathrm{CO}_{2} / \mathrm{Xe}$ gas separation, to examine $\mathrm{Kr} / \mathrm{Xe}$ gas separation via DD3R zeolite membrane, to understand and explain the reported experimental trends, and to describe the mechanisms of such separations at the molecular level, along with the effects of pressure and temperature on gas permeation.

Zeolites are available in a wide range of pore size distributions. DDR3 has been identified as a potential candidate for the separation of $\mathrm{Xe} / \mathrm{Kr}$. Our results have agreed with all trends reported experimentally. Simulations use much smaller membrane thicknesses than actual experiments and are on a much smaller time scale, so there is no realistic expectation of simulation results numerically agreeing with experimental results. The main goal of simulations once the trends agree is to understand the mechanics of the separations that our simulations were able to establish. Other zeolites have since our work was completed also found to be effective in such separations. A recent paper on chabazite membranes has reported this recently [2].

\section{Materials and Methods}

To obtain comparable results for gas permeation through a porous crystal structure, proper gas density numbers are required to simulate the effects of pressures and/or temperatures for different gas feeds. We carried out 10 nanoseconds (timestep $=1.0$ femtosecond) of NPT ensemble MD simulation of only gas atoms, for pure and equimolar mixture, $\mathrm{Kr}$, and $\mathrm{Xe}$ to estimate gas density numbers under different temperatures and pressures.

Gas densities obtained from NPT simulations were used to pack the feed region of the DD3R gas permeation system with the correct number of gas atoms to simulate desired conditions. A typical system set-up is shown in Figure 1. Gas atoms were packed into the central region in Figure 1 using the PACKMOL, which provides a non-overlapping initial configuration [7]. Two DD3R zeolite membrane slabs of thickness $25 \AA$ are used to separate gas feed in the central region, while the two vacuum regions on both edges of normal to the membrane surface serve as the drive force to induce the gas permeation. 
This makes the simulation system compatible with the periodic boundary conditions used. The membrane structure was obtained from the Database of Zeolite Structures [8]. This method of nonequilibrium MD simulation has been implemented successfully to study mass transfer through porous mediums in several applications, such as ion-exchange, alcohol dehydration, reverse osmosis, and gas separations [9-12]. These references detail the specific considerations involved in setting up systems of such simulations.
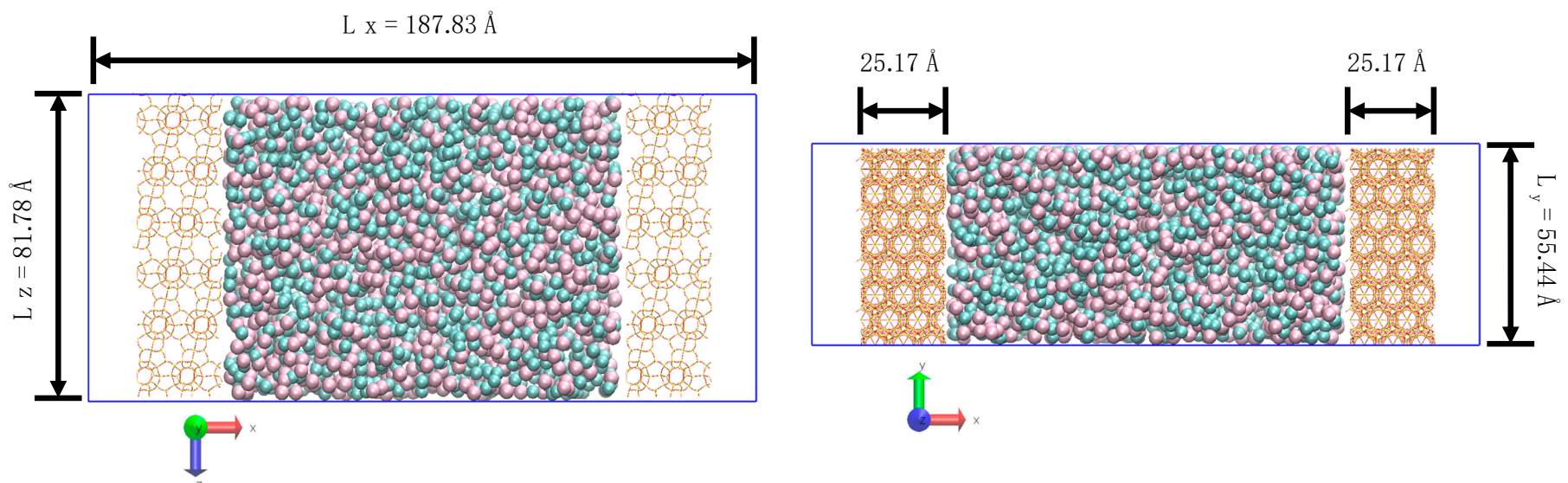

Figure 1. Overall view of the simulation box including top view (left) and front view (right) at the beginning of the simulation. The membrane is $2.517 \mathrm{~nm}$ thick.

As shown in Figure 2, the DD3R unit cell is trigonal; the way we truncated the crystal structure leaves open cages on the surface. These open cages act as the surface adsorption sites for gas atoms. These openings end with inner cage apertures. The smaller cages, with 5-member ring windows shown in Figure 3a, are not accessible to guest atoms such as $\mathrm{Kr}$ and $\mathrm{Xe}$. The 8-member ring window of the alpha cages in Figure $3 \mathrm{~b}$ is $3.6 \AA$ by $4.4 \AA$ representing the VDW distances between oxygen atoms [8], resulting in a barrierheight-limited $\mathrm{Xe}($ size $=4.1 \AA$ ) permeation, a condition that is required for a high $\mathrm{Kr} / \mathrm{Xe}$ separation factor, as we shall see later.
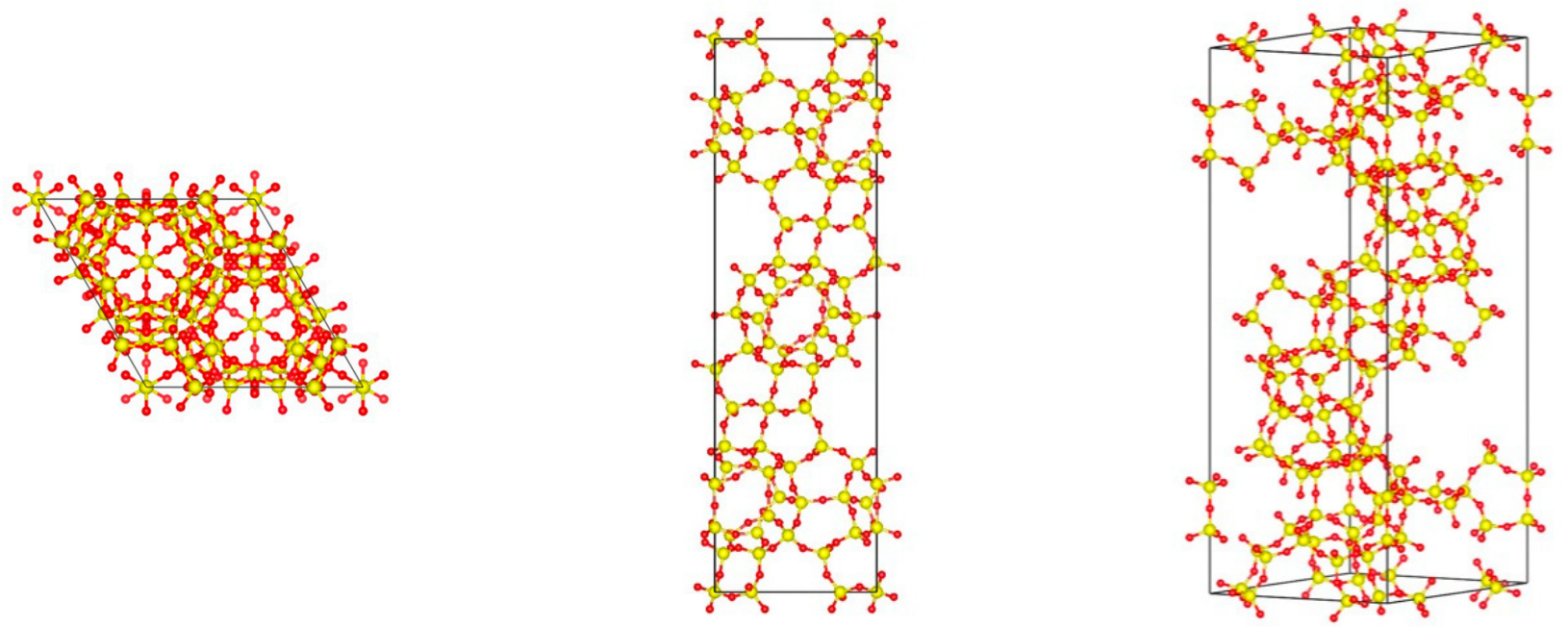

Figure 2. Visualization of DD3R framework unit cell. 


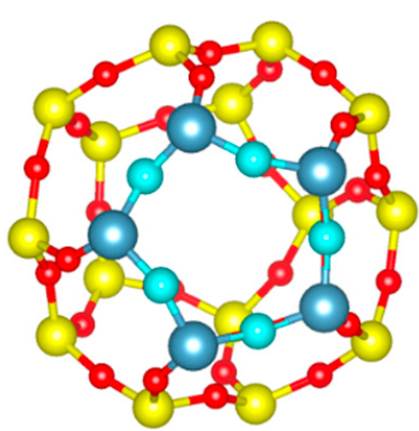

(a)

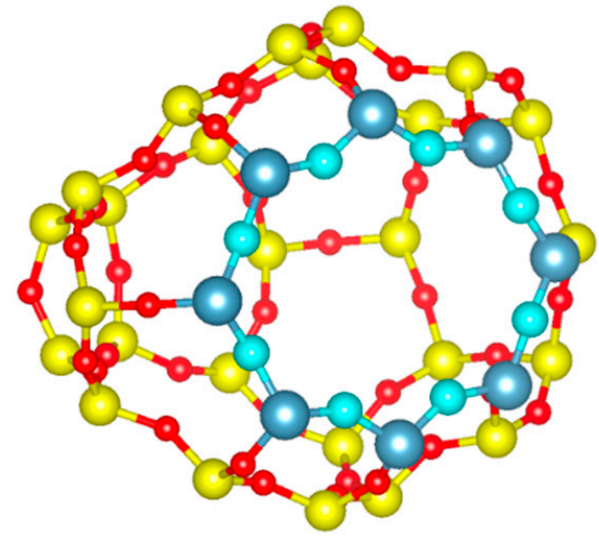

(b)

Figure 3. Visualization of the different cages of DD3R: (a) Visualization of DD3R sodalite cage. Aperture atoms are in different colors for visualization purposes (oxygen is cyan and silicon is silver); (b) Visualization of the DD3R alpha cage, showing the 8-member ring opening with the smallest interatomic distance between two opposing oxygens of $(3.6 \AA)$.

Using the LAMMPS MD software package [13], we performed 30 ns NVT ensemble MD simulations to study the effect of pressure, temperature, and pure vs. mixture feed on gas permeation. With temperature and volume being fixed for the whole duration of each run, the number of gas atoms required to simulate the desired conditions was calculated from density numbers obtained from the NPT MD simulations explained earlier. All gas permeation simulations used a timestep of 1.0 femtosecond.

We used the 12-6 Lennard-Jones potential for inter-molecular interactions. The 12-6 Lennard-Jones potential is given by (1), where $\varepsilon$ is the depth of the potential well and $\sigma$ is the inter-atomic distance at which potential is equal to zero. The parameters are detailed in Table 1. Lorentz-Berthelot mixing rules were used for interactions of cross-species. We considered a global cut-off distance of $12 \AA$ for the potential interaction. In our studies, we assumed a rigid structure for the zeolite. Pictures of crystalline structure were rendered by VESTA [14], while other molecular visualizations, were done using VMD (Visual Molecular Dynamics software) [15].

$$
\begin{aligned}
V_{i j}=4 \varepsilon_{i j}\left[\left(\frac{\sigma_{i j}}{r_{i j}}\right)^{12}-\left(\frac{\sigma_{i j}}{r_{i j}}\right)^{6}\right] \quad r_{i j}<r_{c u t-o f f} \\
\varepsilon_{i j}=\sqrt{\varepsilon_{i} \varepsilon_{j}} \\
\sigma_{i j}=\frac{\sigma_{i}+\sigma_{j}}{2}
\end{aligned}
$$

Table 1. 12-6 Lennard-Jones potential parameters.

\begin{tabular}{cccc}
\hline Pair & $\mathcal{\varepsilon}(\mathbf{k c a l} / \mathbf{m o l})$ & $\boldsymbol{\sigma}(\AA)$ & Reference \\
\hline $\mathrm{Si}-\mathrm{Si}_{\text {(zeolite) }}$ & 0.0010 & 1.000 & {$[4]$} \\
$\mathrm{O}_{\text {(zeolite) }}$ & 0.1898 & 3.000 & {$[4]$} \\
$\mathrm{Kr}_{\text {-Kr }}$ (gas) & 0.3380 & 3.690 & {$[16]$} \\
$\mathrm{Xe}^{-\mathrm{Xe}_{\text {(gas) }}}$ & 0.4190 & 4.100 & {$[16]$} \\
\hline
\end{tabular}

Data analyses include number densities of each species in different regions, and calculation of running averages of these values over the duration of the simulation. Running averages were calculated simultaneously during the MD. These averages were collected 
after having allowed the system to equilibrate for 1 microsecond. In addition, density profiles along the direction normal to the membrane surface facing the gas are plotted by using the running average of the number of atoms in each bin (bin width $=1 \AA$ ), along the $x$-axis. Density profiles are normalized by the total number of atoms of the same species. Density profiles exhibit almost two occupancy peaks in each layer of DD3R cages. These correspond to the favorable adsorption positions within each cage along the permeation path. The numerical results and discussions of density profiles are supported by molecular level visualizations, which were obtained by reading the molecular dynamics trajectories of each run using VMD. The observed selectivity of the $\mathrm{Xe} / \mathrm{Kr}$ systems depends upon both the sizes of the atoms as well as their molecular interactions with the zeolite. These are usually referred to as steric and dispersive attraction effects. The attractive forces lead to one atom finding a more hospitable environment for adsorption on the surface as well as the cavities of the zeolite.

While all simulations span $30 \mathrm{~ns}$ each, for $\mathrm{Xe}$, no complete permeation nor presence in inner cages was ever observed under any simulation conditions considered. Therefore, further examination of the adsorption layer at the membrane surface was helpful in determining the saturated conditions in this region. Two-dimensional density plots on yz plane, for the two adsorption layers formed by Xe in addition to the first layer of open cages, were prepared to examine the membrane surface for active adsorption sites. Another fact related to Xe not completely permeating, is its possible effect on the $\mathrm{Kr}$ permeation; that is, Xe forms concentrated adsorption layers that might act as a barrier to $\mathrm{Kr}$ in the case of mixture simulations. To address these problems, DD3R cages were prefilled with Xe ahead of starting the MD runs for the gas mixture. This prefilling follows a manual scheme where each inner cage is populated with its maximum occupancy of two Xe atoms ahead of starting the simulation.

\section{Results}

We present here the numerical results from running averages obtained from counting the number of gas atoms in each compartment of the simulation box obtained from our MD simulations and reported in Table 2. In addition, density profiles obtained from chunk calculations in these MD simulation as well as visualization of portions of individual snapshots (single time frame) taken from MD trajectories are presented.

Table 2. Numerical results of 30 ns NVT gas permeation system *.

\begin{tabular}{|c|c|c|c|c|c|c|}
\hline Gas Composition & No. & Pressure & Temperature & Feed Region & Membrane Region & Complete Permeation \\
\hline \multirow{3}{*}{ Pure Kr } & (1) & $75 \mathrm{~atm}$ & $300 \mathrm{~K}$ & $0.7302(878)$ & $0.2595(312)$ & $0.0138(17)$ \\
\hline & (2) & $150 \mathrm{~atm}$ & $300 \mathrm{~K}$ & 0.8225 (1977) & $0.1650(396)$ & $0.0116(28)$ \\
\hline & (3) & $150 \mathrm{~atm}$ & $425 \mathrm{~K}$ & $0.7940(1081)$ & $0.1684(229)$ & $0.0369(50)$ \\
\hline \multirow{3}{*}{ Pure Xe } & (4) & $75 \mathrm{~atm}$ & $300 \mathrm{~K}$ & $0.9478(1442)$ & $0.0518(155)$ & \\
\hline & (5) & $150 \mathrm{~atm}$ & $300 \mathrm{~K}$ & $0.9019(3030)$ & $0.0972(166)$ & \\
\hline & (6) & $150 \mathrm{~atm}$ & $425 \mathrm{~K}$ & $0.9232(1364)$ & $0.0762(113)$ & \\
\hline $\mathrm{Kr}$ in $\mathrm{Kr}-\mathrm{Xe}$ Mixture & (7) & $150 \mathrm{~atm}$ & $300 \mathrm{~K}$ & $0.8512(1198)$ & $0.1394(196)$ & $0.0088(12)$ \\
\hline Xe in $\mathrm{Kr}-\mathrm{Xe}$ Mixture & (7) & $150 \mathrm{~atm}$ & $300 \mathrm{~K}$ & $0.9188(1293)$ & $0.0804(113)$ & \\
\hline $\begin{array}{l}\mathrm{Kr} \text { in } \mathrm{Kr}-\mathrm{Xe} \text { Mixture } \\
\text { (Xe Pre-filled DD3R) }\end{array}$ & (8) & $150 \mathrm{~atm}$ & $300 \mathrm{~K}$ & $0.8916(1255)$ & $0.0838(118)$ & $0.0240(34)$ \\
\hline $\begin{array}{l}\text { Xe in Kr-Xe Mixture } \\
\text { (Xe Pre-filled DD3R) }\end{array}$ & (8) & $150 \mathrm{~atm}$ & $300 \mathrm{~K}$ & $0.9189(1294)$ & $0.0803(113)$ & \\
\hline
\end{tabular}

* Numerical fractions are calculated based on the running average of the number of atoms of each individual species divided by the total number of its atoms in the different regions of the system. Actual number of atoms is within parentheses. 


\subsection{Pure Gases}

\subsubsection{Effect of Pressure on Pure Kr Permeation}

From Figure 4, it is observed that, from low to high pressure, more krypton atoms accumulate at the surface of the membrane facing the gas feed region; this is a direct consequence of the higher number density at the higher pressure. We note, however, that the ratio of the total number of adsorbed $\mathrm{Kr}$ atoms (integral over the adsorption peak) from 150 to $75 \mathrm{~atm}$ is 1.71, which is less than 2; the greater adsorption at the higher pressure is attributed to the higher driving force. $\mathrm{Kr}$ permeation into the membrane is also greater at the higher pressure. Inside the membrane, the peaks in Figure 4 correspond to occupation of cages within the membrane as the $\mathrm{Kr}$ atoms permeate. There are only small differences in these peaks at the two pressures. Given the membrane structure, cages have a finite capacity, thus, the pressure effect toward increasing krypton content inside the membrane can be limited when cages become saturated. A closer look into the inner cages is illustrated in Figure 5, which shows the maximum number of $\mathrm{Kr}$ atoms observed in a single cage was 4 for both pressures, confirming the incidence of saturated cages. Overall, higher pressure yields higher krypton permeation and adsorption. The pressure effect on adsorption for many similar surfaces has been extensively studied by many and the results obtained almost always show a linear dependence on pressure [1,2]. We therefore felt that studying two pressures was sufficient since our primary interest was in obtaining the pressure gradient, which can be obtained from two points sufficiently apart $[17,18]$.

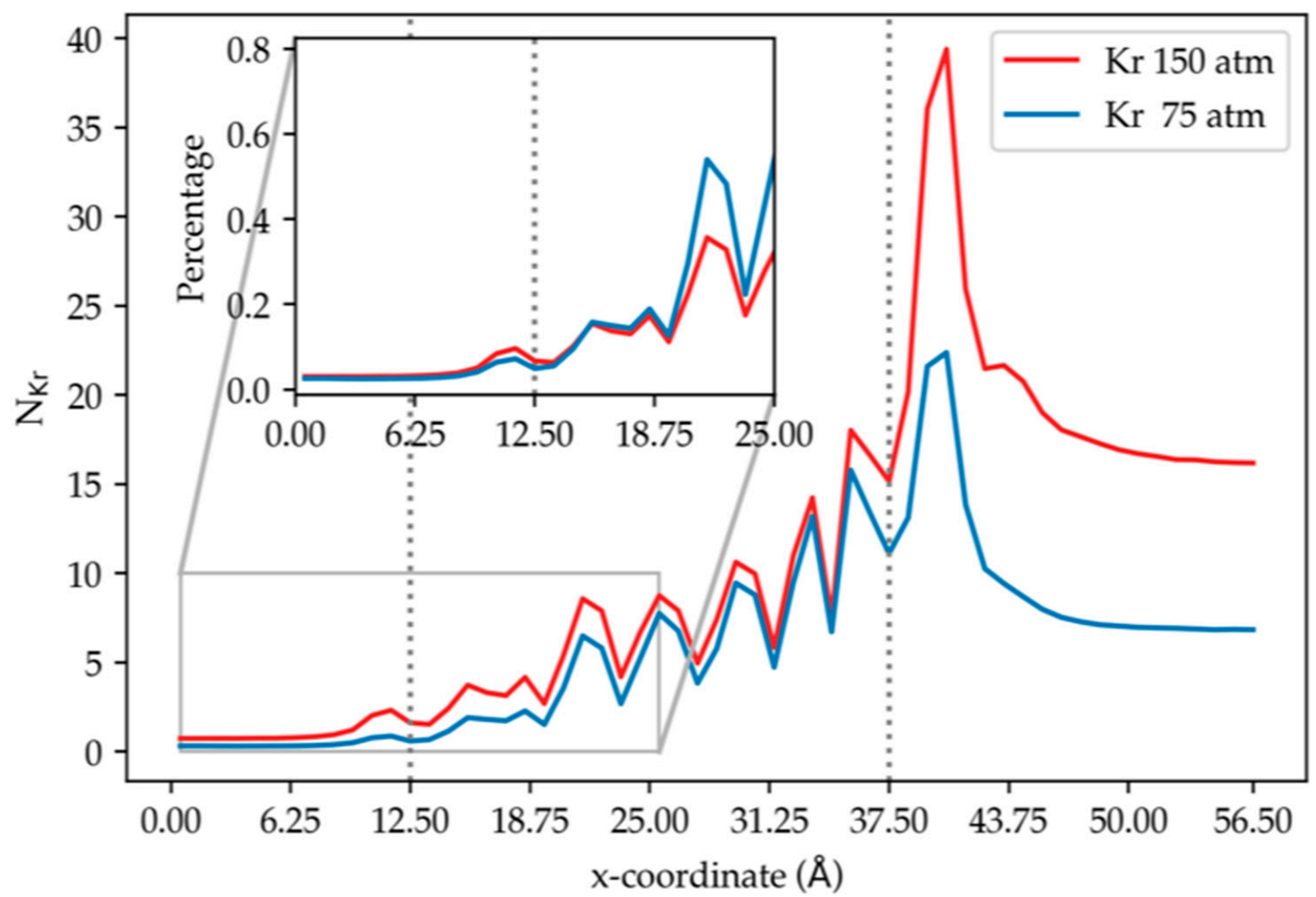

Figure 4. Density profiles of krypton along the direction normal to membrane surface (bin width $=1 \AA$ ). $\mathrm{Ni}=$ running average of the number of $\mathrm{Kr}$ atoms in the ith bin using results from simulation (1) and (2) at $300 \mathrm{~K}$. The dashed vertical lines correspond to the membrane edges. For this and succeeding figures, the profiles in only one of the membranes in the simulation box are shown, since the other is related by symmetry. 


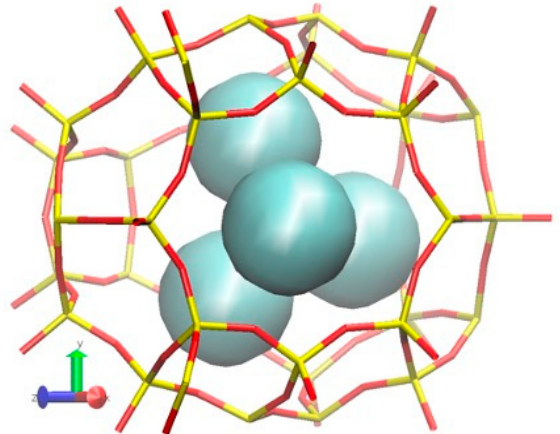

(a)

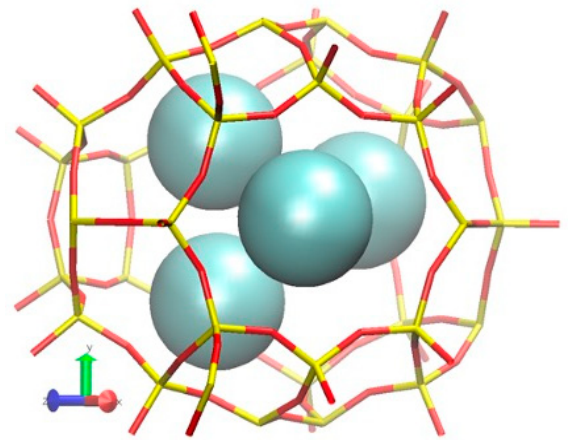

(b)

Figure 5. Molecular visualization of $\mathrm{Kr}$ atoms filling up inner cages of DD3R membrane using molecular trajectories from simulations (a) at $300 \mathrm{~K}$ and $150 \mathrm{~atm}$ and (b) at $300 \mathrm{~K}$ and $75 \mathrm{~atm}$.

\subsubsection{Effect of Temperature on Pure Kr Permeation}

At the lower temperature, it is observed in Figure 6 that more $\mathrm{Kr}$ atoms adsorbed on the DD3R surface than at the higher temperature. The integrated adsorption peak on the DD3R surface at the lower temperature is twice as large as at the higher temperature. We note that while there is a secondary adsorption layer at $300 \mathrm{~K}$ (already discussed in Figure 4), there is no second adsorption layer at the higher temperature. This is because at the higher temperature, $\mathrm{Kr}$ can overcome the energy barriers and enter the cages, causing less accumulation of $\mathrm{Kr}$ at the surface. The rate of desorption from inner cages is higher at the high temperature than at the low temperature, allowing fewer krypton atoms to accumulate inside the membrane cages as exhibited in Figure 7a,b. This is explained by the increased gas-particle velocity at the high temperature, which overcomes intermolecular energies between krypton and DD3R cages, thereby promoting higher incidence of complete krypton permeation as shown in Figure 7c,d. By comparing simulations (2) and (3), we find that increasing temperature has tripled the ratio of the completely permeated krypton atoms relative to the total number of krypton atoms. We would also like to point out that experimental behavior of adsorption of noble gases on a range of surfaces show an almost linear behavior w.r.t. temperature as long as the temperature range is not exceeding wide. For example, a recent study in the $77-150 \mathrm{~K}$ range for nitrogen, showed essentially a linear variation with temperature for a range of pressures [19].

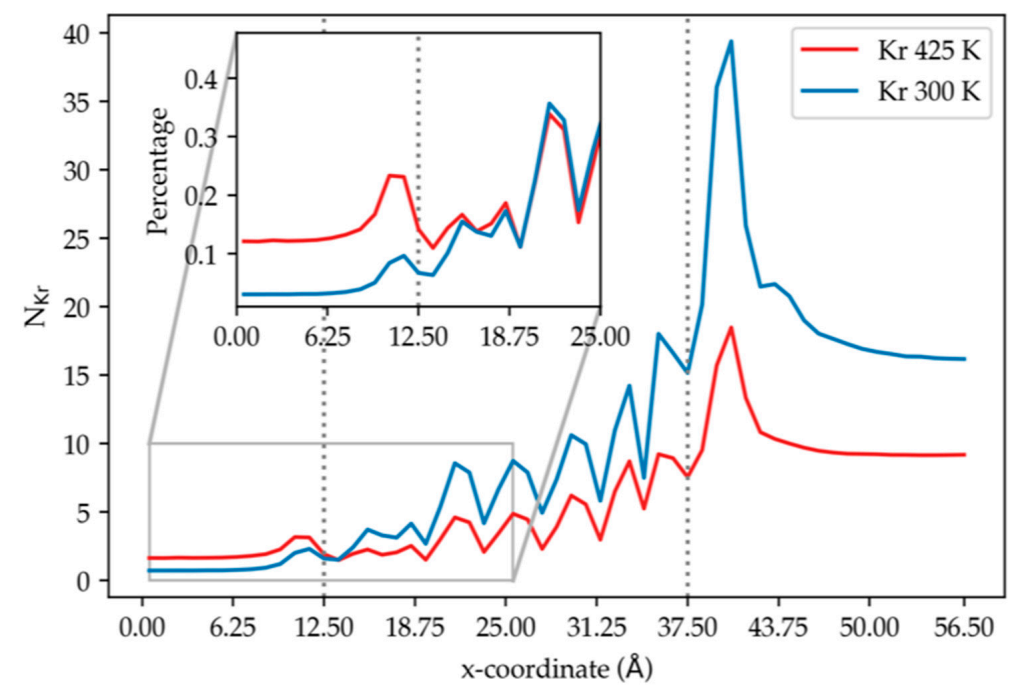

Figure 6. The density profile of krypton at different temperatures, normalized by the total number of atoms of the same species in the system, along the direction normal to the membrane surface, using results from simulation (2) and (3) at $150 \mathrm{~atm}$. The dashed vertical lines correspond to the membrane edges. 


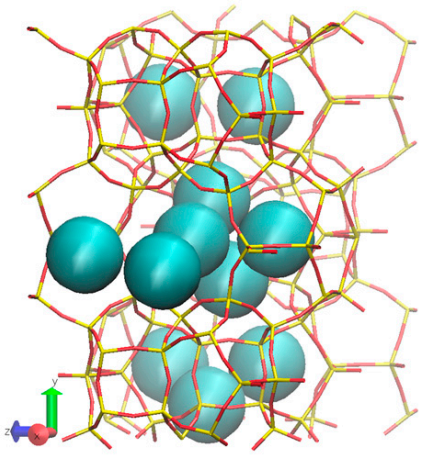

(a)

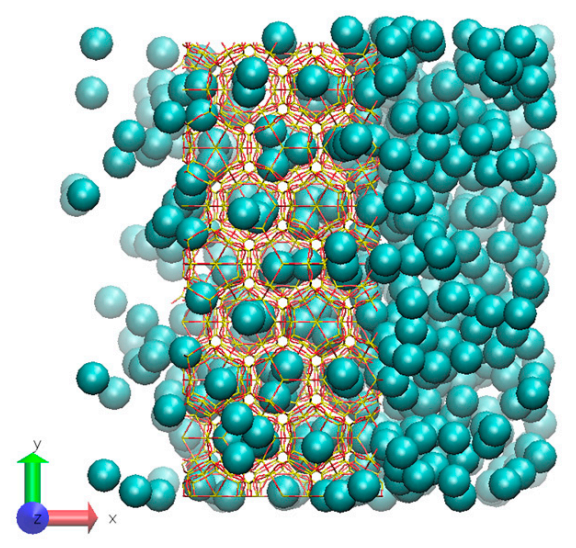

(c)

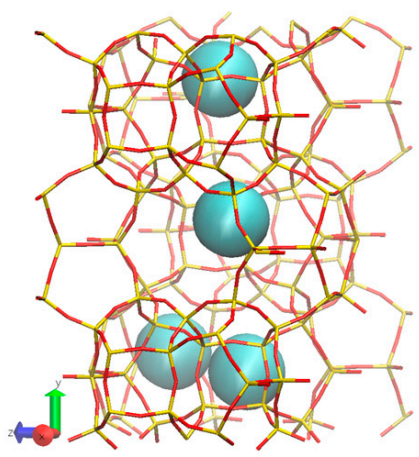

(b)

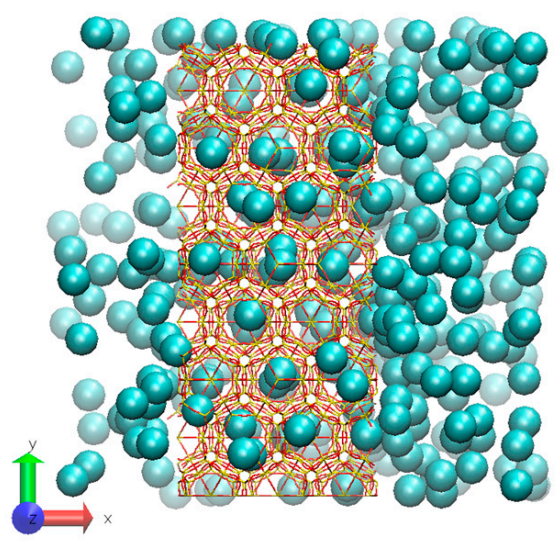

(d)

Figure 7. Molecular visualization of the temperature effect: (a) (inner cages) $300 \mathrm{~K}$ and 150 atm; (b) (inner cages) $425 \mathrm{~K}$ and $150 \mathrm{~atm}$; (c) (overall view) $300 \mathrm{~K}$ and $150 \mathrm{~atm}$; (d) (overall view) $425 \mathrm{~K}$ and $150 \mathrm{~atm}$.

\subsubsection{Effect of Pressure on Pure Xe Permeation}

We note that, under the same pressure, Xe has higher number density in comparison to $\mathrm{Kr}$; Xe gas thus behaves less ideally than $\mathrm{Kr}$. At both pressures, complete permeation of Xe through the DD3R zeolite membrane has not been observed. However, the observation of the first sharp peak inside the membrane indicates that Xe atoms can and do enter the membrane, although less easily than $\mathrm{Kr}$, and some permeation will be observed after much longer simulation times. Figure 8 illustrates how the adsorption layers at both pressures are relatively close, indicating saturation of active sites on the membrane surface. This observation implies that the size of $\mathrm{Xe}$ atom relative to the diameter of the structure openings into the cages of the membrane creates a barrier-height-limited permeation. At the membrane surface, the Xe adsorption peak at higher pressure is greater than that at the lower pressure, as was found in the case of pure Kr. However, the ratio of the integrated peak intensity at $150 \mathrm{~atm}$ to that at $75 \mathrm{~atm}$ is 1.27 , compared to 1.71 for the pure $\mathrm{Kr}$. This indicates that, unlike the $\mathrm{Kr}$ case, the Xe saturates the membrane surface, a consequence of the greater attractive Xe-membrane interactions compared to Kr-membrane, as well as greater attractive $\mathrm{Xe}-\mathrm{Xe}$ interactions compared to $\mathrm{Kr}-\mathrm{Kr}$. In addition, at higher pressures, Xe has a second peak in the density profile away from the membrane. This second adsorption layer forms primarily due to Xe-Xe interactions of the Xe atoms in the feed region with the ordered Xe first adsorption layer. 


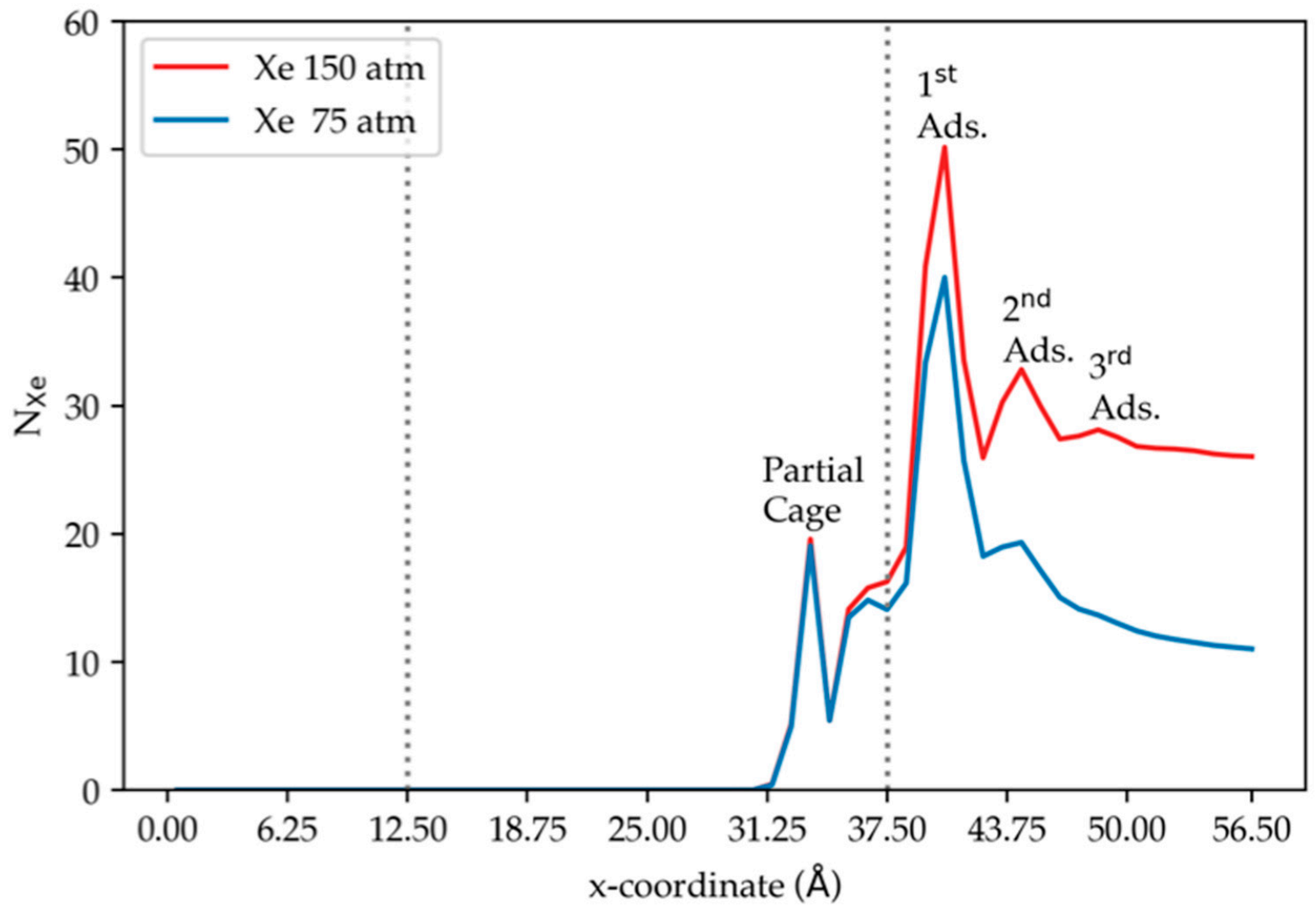

Figure 8. The density profile of xenon along the direction normal to the membrane surface at two pressures, using results from simulation (4) and (5) at $300 \mathrm{~K}$. The dashed vertical lines correspond to the membrane edges.

To further investigate this accumulation within the first adsorption layer on the membrane surface, two-dimensional density plots were constructed to examine if Xe adsorption exhibits any specific structure that may provide insight into active adsorption sites on the DD3R membrane surface. Figure 9 examines the adsorption of Xe on the membrane surface (in yz-plane). The three sets of the two-dimensional density plots illustrate Xe distribution on the surface for the three peaks shown on the bottom panel of the figure. The first row represents the second (outer) adsorption layer under two pressures, where the left panel has a darker shade indicating that the second adsorption layer starts to saturate under high pressure.

The second row of plots examines the surface adsorption layer. It is observed that the higher-pressure results in higher adsorption of Xe on the membrane surface than the low-pressure. This can be explained by the higher driving force pushing Xe closer to the membrane in high numbers, where they accumulate on the surface, given the strong intermolecular interaction of Xe with the zeolite membrane. The Xe arrangement on the surface provides locations of active adsorption sites on the membrane surface in contact with fluid. The third row of plots examines the inner adsorption layer of Xe in the partly open cages at the surface of the DD3R membrane. The two plots are similar, which agrees with the bottom panel showing the identical density profiles. Due to the barrier-heightlimited entry into cages and Xe-cage intermolecular interactions, Xe has shown a much slower permeation process in comparison with $\mathrm{Kr}$. 

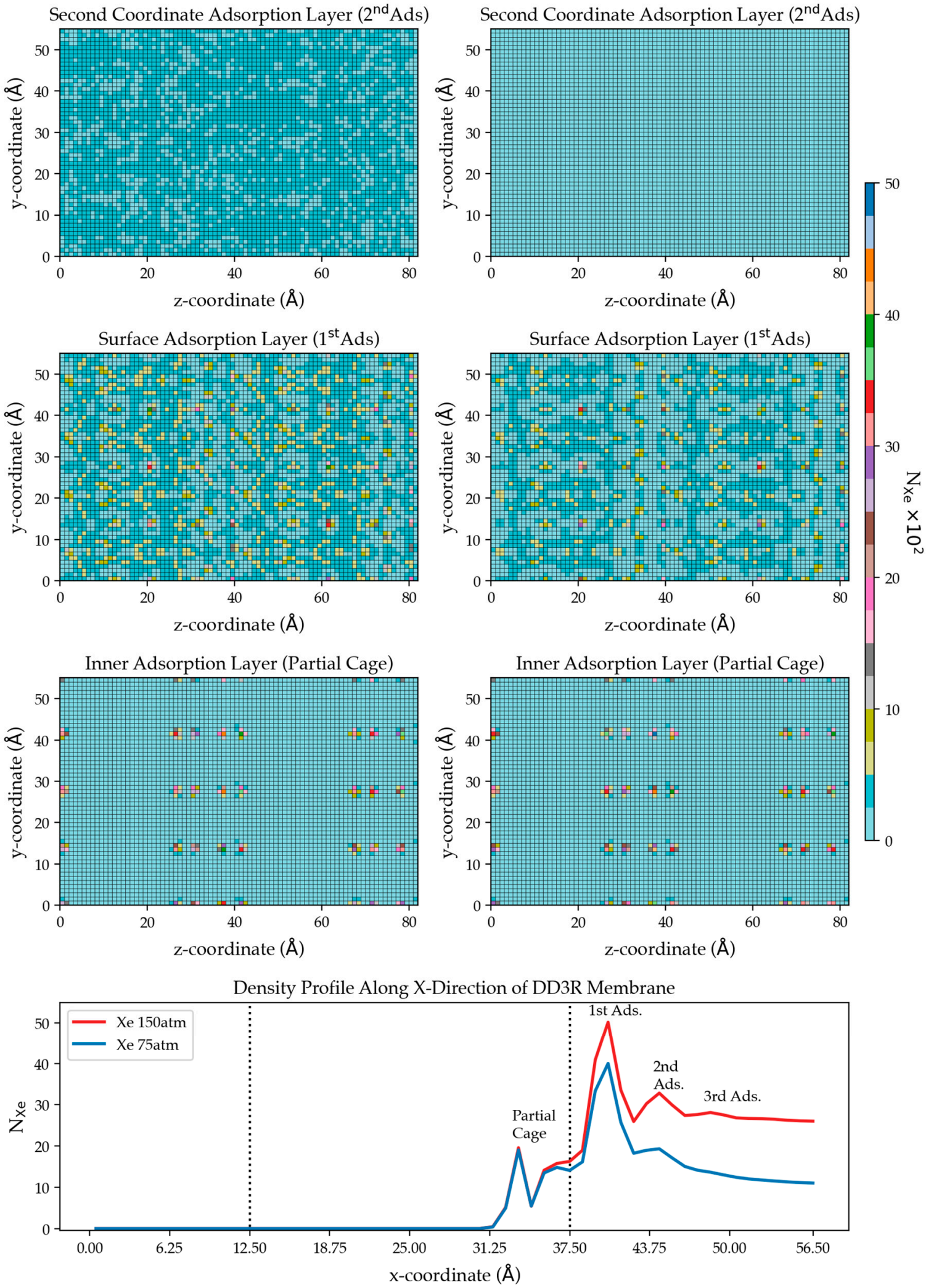

Figure 9. Two-dimensional density plots illustrating adsorption of $\mathrm{Xe}$ atoms on the membrane surface at two pressures using results of simulation (4) $75 \mathrm{~atm}$ (right) and (5) $150 \mathrm{~atm}$ (left). The top 2 plots correspond to the number density from the integrated peak of the secondary adsorption layer in Figure 8, followed by that of the surface adsorption peak, and the prominent peak inside the membrane. The bottom plot is a copy of Figure 8 as a reminder. 


\subsubsection{Effect of Temperature on Pure Xe Permeation}

Figure 10 shows the density profiles under different temperatures. At low temperature, Xe atoms accumulate more for all adsorption layers. Lower Xe occupancies are found at open cages on the membrane surface, in which the cages have partial shapes with much larger opening than the 8-member DD3R window. This is illustrated in Figure 11 showing average occupancy for partial cages decreasing to two Xe atoms at the most for the majority of the MD runs. No complete permeation takes place even at $425 \mathrm{~K}$ for the length of MD simulations considered in this study. Figure 11a shows Xe atoms occupying an inner cage Figure $11 \mathrm{~b}$ becomes reduced at a much elevated temperature, while Figure 11c Xe atoms inner cage occupancy has been observed at a much elevated temperatures at similar simulation times.

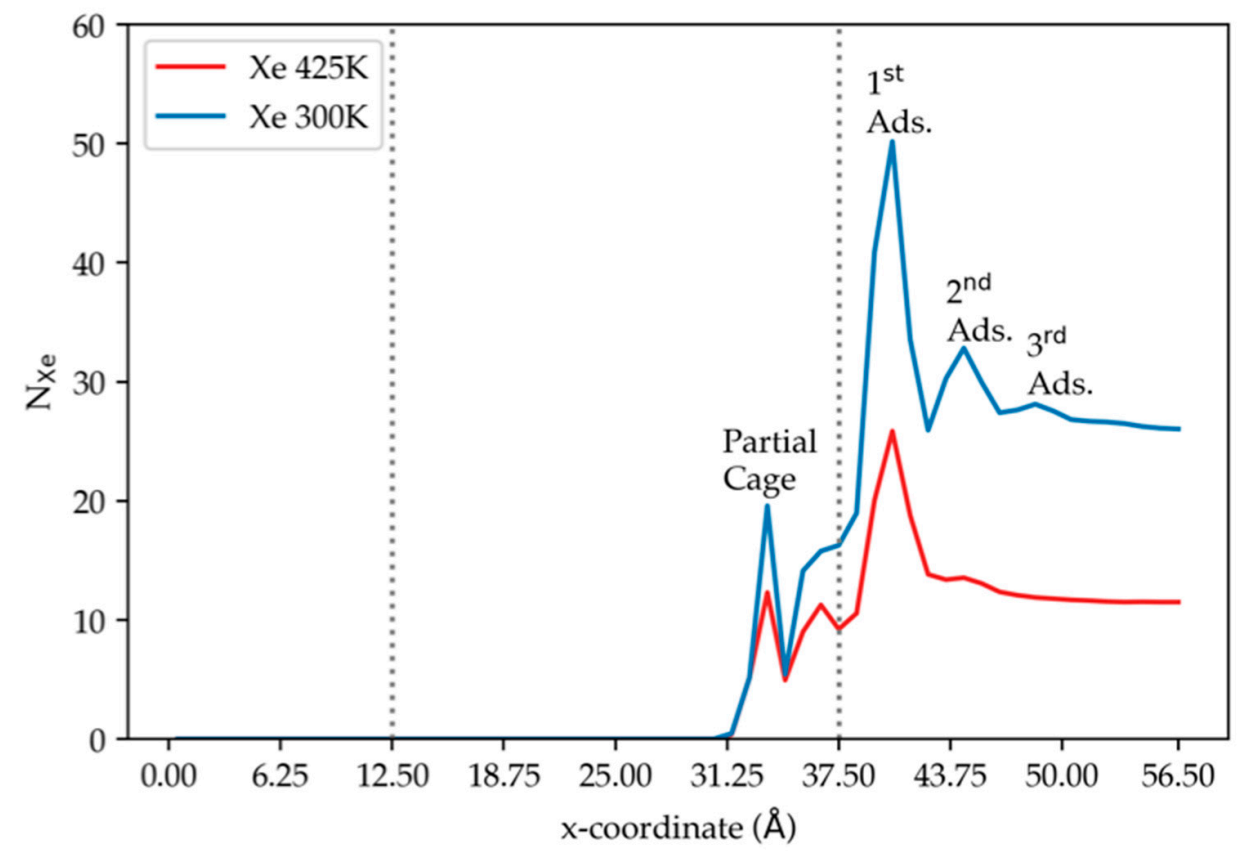

Figure 10. The density profile of xenon along the direction normal to the membrane surface at two pressures, using results from simulation (5) and (6) at $150 \mathrm{~atm}$. The dashed vertical lines correspond to the membrane edges.

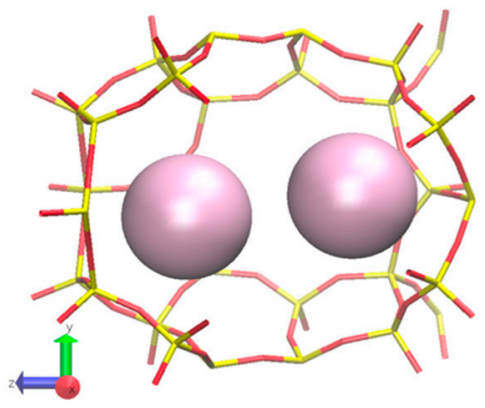

(a)

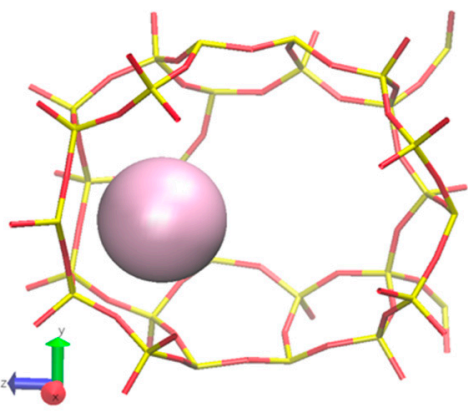

(b)

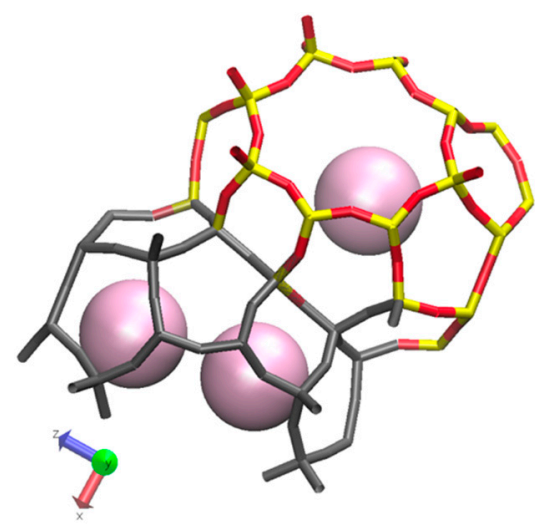

(c)

Figure 11. Molecular visualization of temperature effect on active adsorption site occupancy (a) at $300 \mathrm{~K}$ and $150 \mathrm{~atm}$ and (b) at $425 \mathrm{~K}$ and $75 \mathrm{~atm}$. (c) Xe atom occupying inner cages at elevated temperatures validating the requirement of much longer simulation times. Color difference in membrane structure is to distinguish surface partial cages (gray) and inner cages (colored). 


\subsection{Mixture Gases}

\subsubsection{Kr Permeation in $\mathrm{Kr}-\mathrm{Xe}$ Mixture}

Figure 12 illustrates the density profile for pure $\mathrm{Kr}$ and for $\mathrm{Kr}$ in equimolar $\mathrm{Kr}-\mathrm{Xe}$ mixture along the $\mathrm{x}$-direction on the membrane surface in contact with the gas. As shown in the figure and discussed earlier, in pure $\mathrm{Kr}$ feed, $\mathrm{Kr}$ atoms accumulate in the adsorption layer at the membrane surface and also in the layer of partial cages at the DD3R surface. In contrast, in the $\mathrm{Kr}-\mathrm{Xe}$ mixture system, the $\mathrm{Kr}$ ratio, at the adsorption layer and in the layer of partial cages, is lower than for the pure $\mathrm{Kr}$ case. This is a direct consequence of the presence of $\mathrm{Xe}$, which is attracted more strongly to the membrane than $\mathrm{Kr}$. The empty DD3R cages at the start of simulation, as shown in Figure 1, are gas atom adsorption sites. The barrier-height-limited permeation of Xe prevents it from entering the cages. So, the Xe atoms accumulating on the surface partial cages prevent $\mathrm{Kr}$ from entering the inner cages and passing through completely, which is contrary to experimental trends. We hypothesize that this can be attributed to the barrier created by Xe atoms, whose adsorption on the membrane surface arises from stronger attractive forces with the membrane. On one side, the kinetic dimeter of $\mathrm{Xe}$ is $4.10 \AA$, which is much larger than $\mathrm{Kr}(3.69 \AA)$ and the pore size of DD3R (3.6 $\AA$ ) [20]. The presence of Xe can effectively block the pathway of $\mathrm{Kr}$ diffusion. Such behavior has been observed in our previous study, where the mixture of $\mathrm{Xe} / \mathrm{CO}_{2}$ has been used in the feed [4]. On the other side, the preferential adsorption of $\mathrm{Xe}$ over $\mathrm{Kr}$ has been proved for various zeolite frameworks experimentally [1,21,22]. If, instead, we start the simulation with DD3R inner cages pre-filled with Xe atoms as shown in Figure 13, we find the percentage of completely permeated $\mathrm{Kr}$ atoms has increased from 1.16 percent in pure $\mathrm{Kr}$ to 2.40 percent in $\mathrm{Kr}-\mathrm{Xe}$ mixture, which agrees with experimental trends [3]. Xe hopping in-between cages would require computational time not currently feasible; on the other hand, pre-filling the cages with Xe mimics the steady-state Xe occupancies at $150 \mathrm{~atm}$, prior to $(\mathrm{t}=0)$ of the MD simulation run with equimolar $\mathrm{Xe}$ and $\mathrm{Kr}$ in the feed. We find that the presence of $\mathrm{Xe}$ in the inner cages assists the $\mathrm{Kr}$ permeation through the membrane because of attractive $\mathrm{Kr}-\mathrm{Xe}$ forces. The membrane cage filling scheme in this MD simulation was achieved by identifying accessible cages and manually filling each with two Xe atoms, which corresponds to steady-state Xe occupancies at $150 \mathrm{~atm}$. Despite this somewhat arbitrary manual filling, the pre-filled Xe atoms quickly find more favorable positions inside the cages.
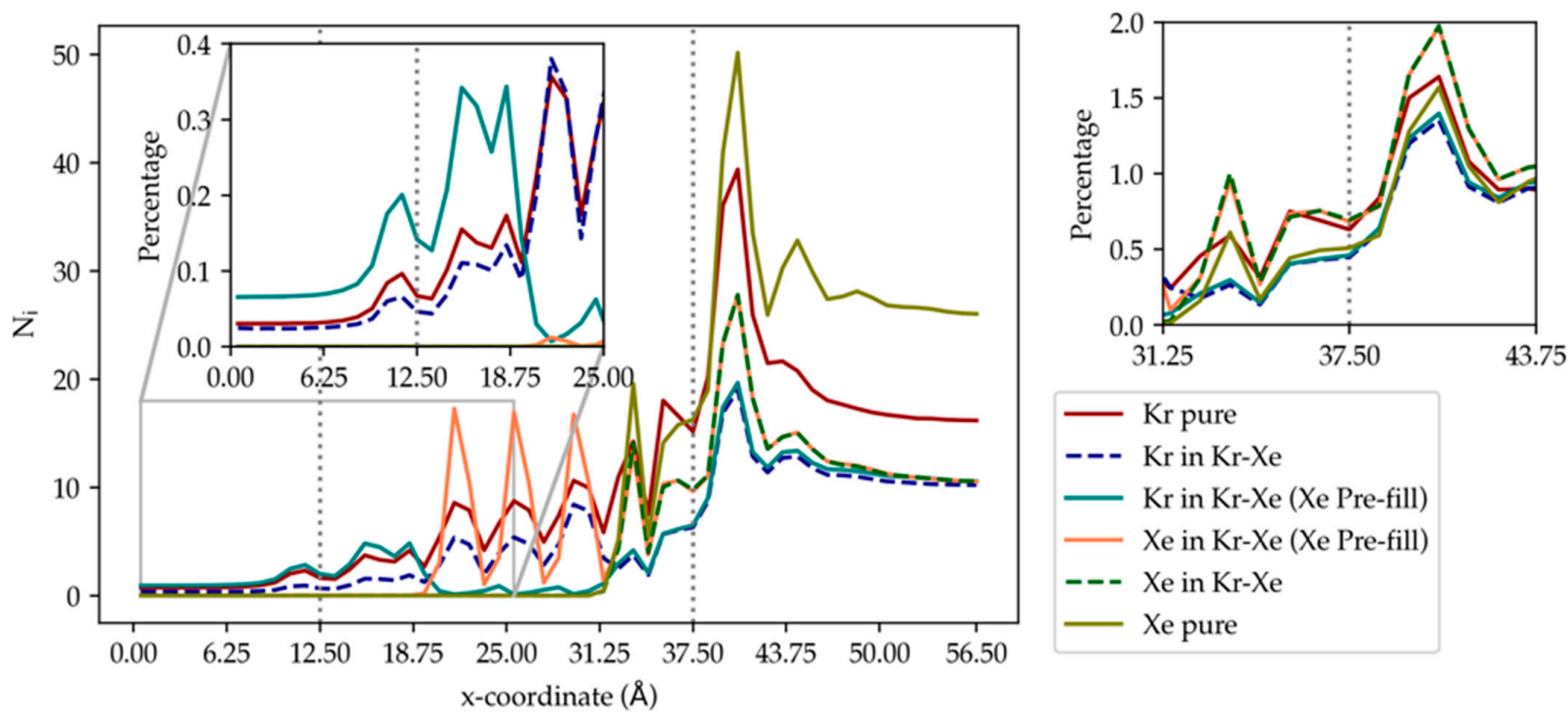

Figure 12. The density profile of $\mathrm{Kr}$ and $\mathrm{Xe}$ along the direction normal to the membrane surface at two pressures, using results from simulation (2), (5), (7), and (8) at $300 \mathrm{~K}$. The dashed vertical lines correspond to the membrane edges. 


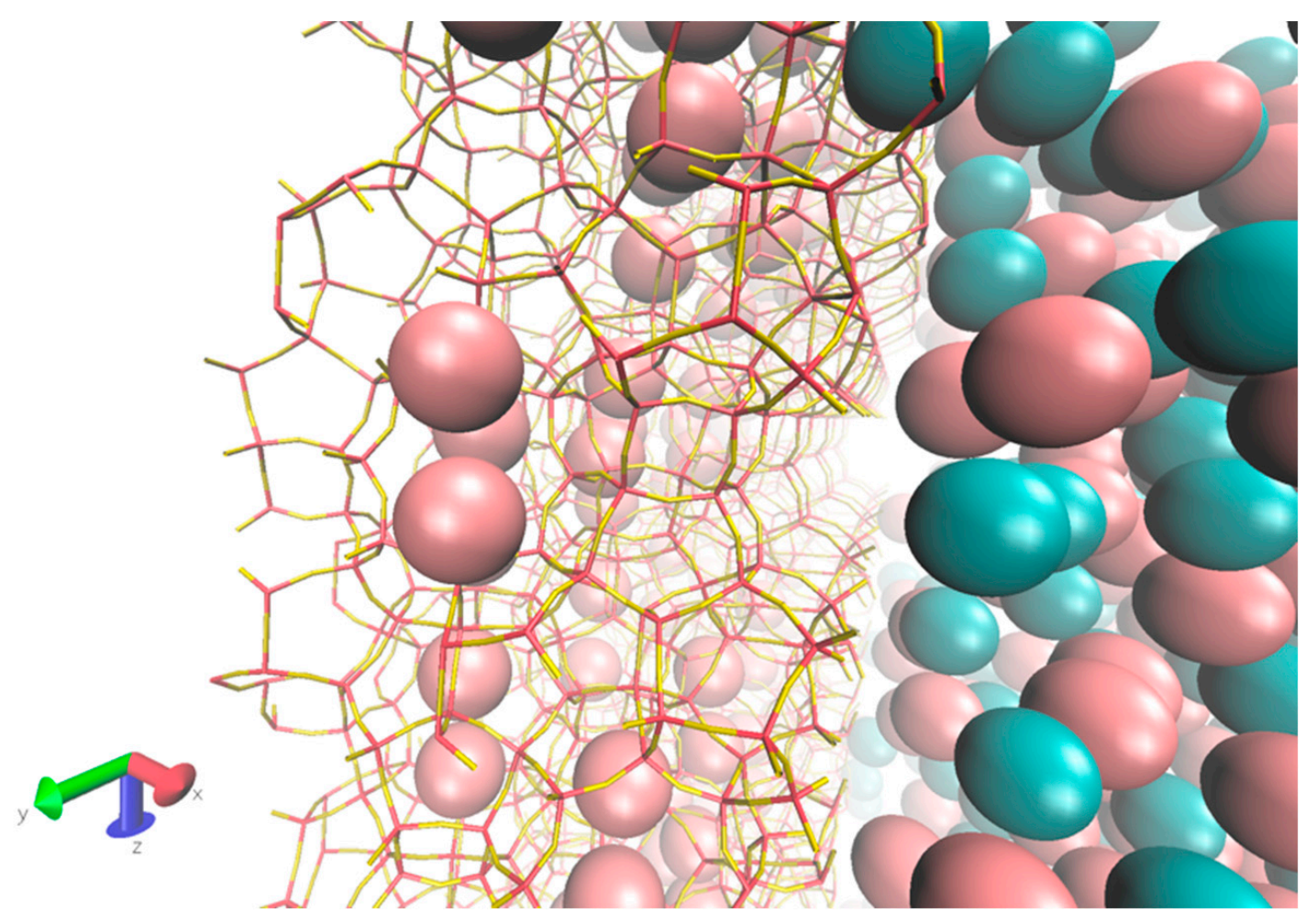

Figure 13. Close-up visualization of the gas-membrane interface at the beginning of the simulation, showing prefilled Xe atoms inside inner cages of DD3R in pink, while $\mathrm{Kr}$ is cyan.

\subsubsection{Xe Permeation in $\mathrm{Kr}-\mathrm{Xe}$ Mixture}

In Figure 12, at the adsorption layer, the highest peak for Xe is close to the membrane surface. The integral over the adsorption peak, expressed as the ratio of adsorbed Xe atoms to the total number of Xe atoms in the system, in the pure case is lower than in the $\mathrm{Kr}-\mathrm{Xe}$ mixture system. This is because of the higher number density of Xe in the pure case. The pure Xe case shows a third small bump away from the membrane, which indicates the saturation of the first two adsorption peaks. Similarly, in the first layer of DD3R partial cages, the profiles show lower ratio for the pure case than the mixture case. Despite greater adsorption in pure $\mathrm{Xe}$, the higher molar density in bulk results in a lower ratio. In addition, the finite number of the partial cages also limits the adsorption capacity. When comparing the actual numbers of Xe atoms in the partial cages layer, the highest peak, the numbers are lower in the mixture case due to the presence of $\mathrm{Kr}$, which has smaller atomic size and could hop in-between cages much more easily. In the case of the DD3R cages pre-filled with Xe atoms, while Xe density profile shown in dashed blue shows a uniform distribution of Xe atoms along the inner cages of the membrane structure, there is no complete permeation of Xe either from the gas feed nor from the Xe atoms initially occupying the inner cages. This validates the assumption on the molecular level in which Xe jumping in and out of cage openings is barrier-limited.

Figure 14 provides a clear visualization of the difference between following the conventional simulation method with empty cages versus using the manual Xe pre-filling scheme discussed earlier. Starting with empty cages, Figure 14a shows Xe forming an adsorption layer on the membrane surface, blocking $\mathrm{Kr}$ permeation given the stronger Xe-membrane interaction. In contrast, pre-filling inner cages with Xe allows for the attractive $\mathrm{Xe}-\mathrm{Kr}$ forces to promote $\mathrm{Kr}$ permeation. As a $\mathrm{Kr}$ atom approaches an inner cage opening, the presence of $\mathrm{Xe}$ in the adjacent cages can attract $\mathrm{Kr}$ and help it passing through the inner cages, which results in higher complete permeation of Kr. Such a permeation mechanism is illustrated in Figure 14b-f. Noting that our hypothesis provided here comes from a modeled system under some ideal circumstances, the observation that $\mathrm{Kr}$ permeation could 
be improved with the presence of Xe has been documented in experimental works for the CHA framework [2].

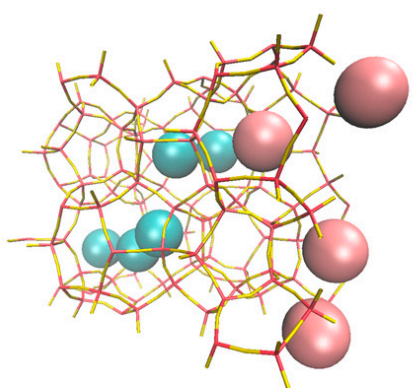

(a)

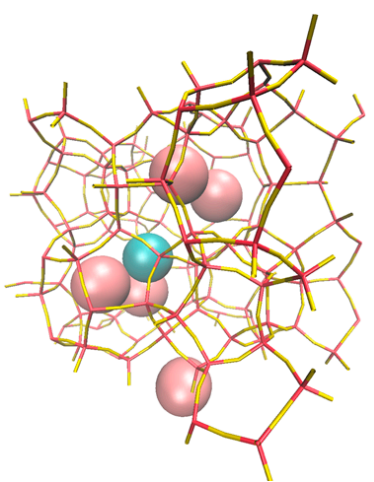

(d)

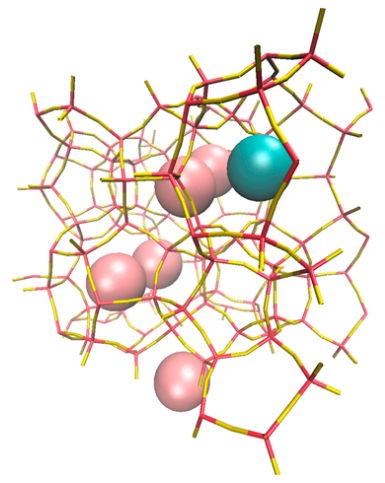

(b)

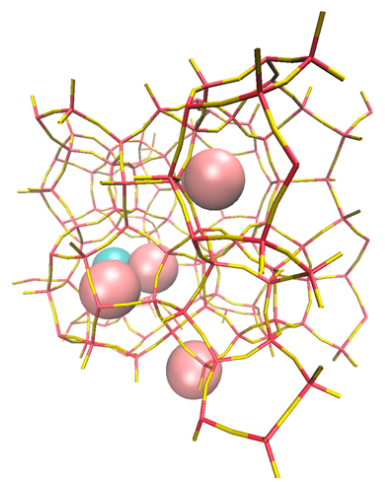

(e)

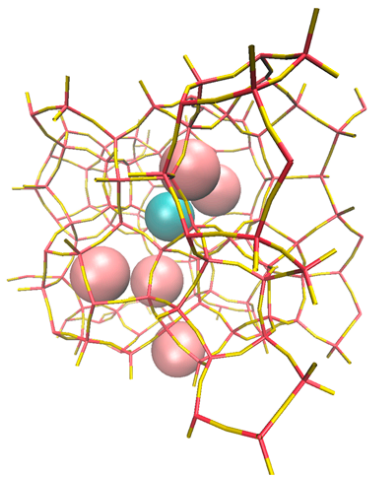

(c)

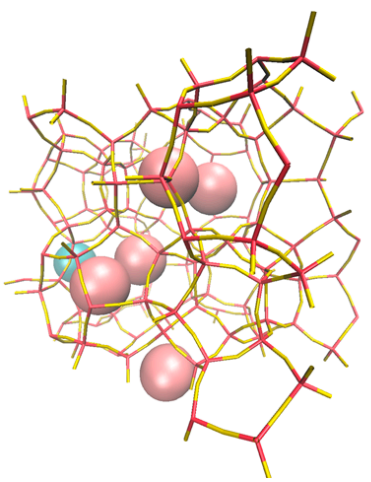

(f)

Figure 14. The absence of Xe pre-filled cages (a) causes Xe to accumulate on the surface, thus limiting $\mathrm{Kr}$ permeation, which, in addition, accumulates inside inner cages. Molecular visualization showing the effect of Xe pre-filled zeolite cages on $\mathrm{Kr}$ permeation, where we see $\mathrm{Kr}$ atom approaches cage window (b), $\mathrm{Kr}$ inside first inner cage (c), $\mathrm{Kr}$ jumping from and into inner cages and passing to the complete permeation region (d), (e), and (f) respectively.

\subsection{MSD Results}

In this computational study, the system is set up with gas feed, where high gas concentration numbers exist, that is trapped between two DD3R zeolite membrane slabs that separate the gas from the complete permeation region where dilute gas concentration numbers exist. This results in a high concentration gradient that acts as the driving force for the gas permeation. As the simulation starts, the gas atoms start to permeate through DD3R membrane pores toward the complete permeation region. During this time, mean square displacements in $\mathrm{x}, \mathrm{y}$, and $\mathrm{z}$ were sampled and averaged over atoms every 10,000 timesteps for the length of each simulation.

Figure 15 illustrates the MSD data, in the direction normal to the membrane surface, plotted against time. The data show the usual fluctuations related to the nature of sampling from molecular dynamics simulations. Data were linearly fit to obtain the slope for each data set for diffusion coefficient calculations. In the direction x-axis, the slope of MSD vs. time is illustrated by Stokes-Einstein Law in Equation (4).

$$
D=\frac{1}{2 n} \frac{\Delta r(t)^{2}}{\Delta t}
$$

With $n$ signifying the number of dimensions and the MSD to time interval ratio as the slope from the data plotted. A summary of diffusion coefficient results for gas permeation 
through DD3R zeolitic membrane is provided in Table 3 for 30 ns of MD simulations at $300 \mathrm{~K}$ and $150 \mathrm{~atm}$.

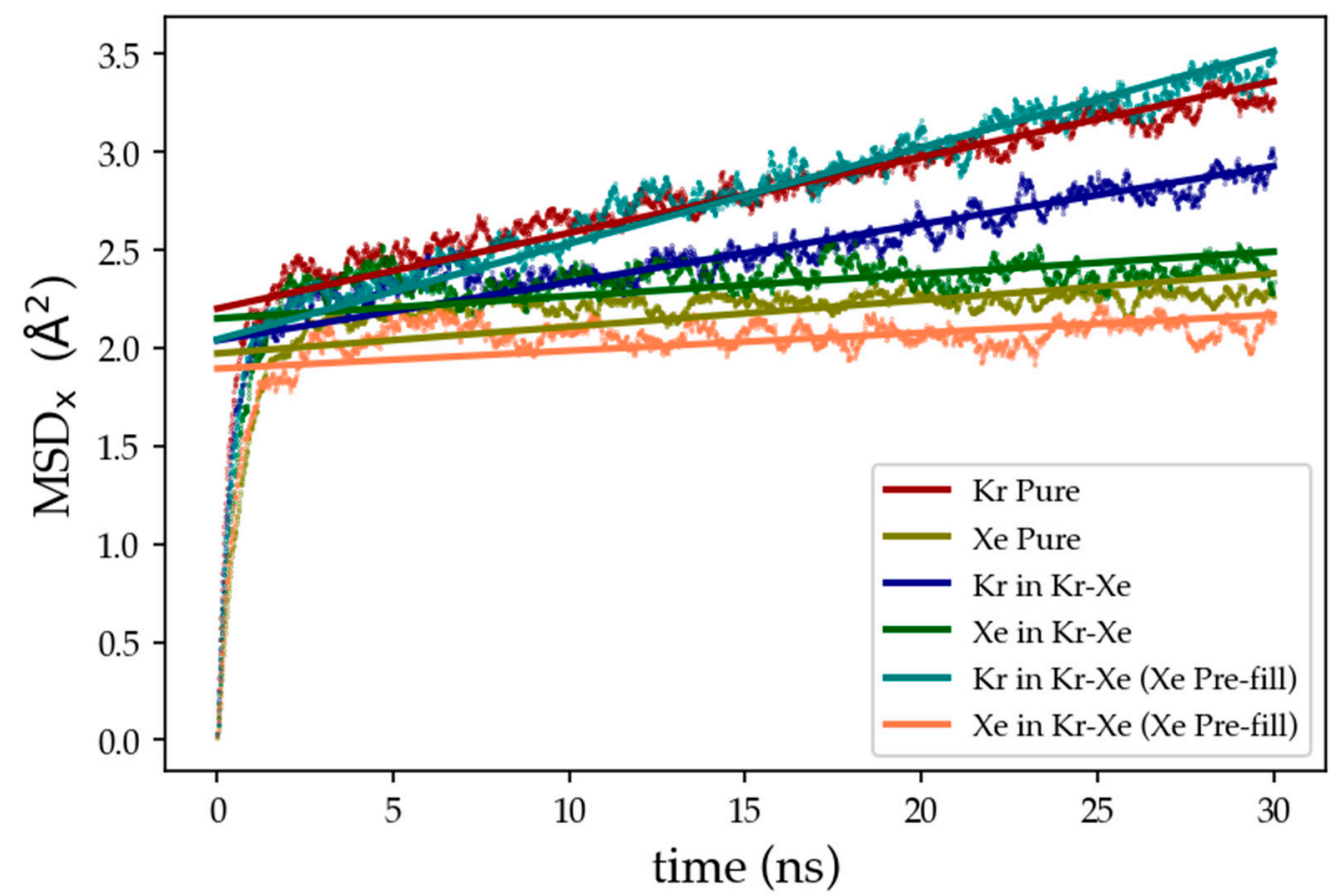

Figure 15. Mean-Squared-Displacement (MSD) in the direction normal to the membrane surface vs. time for $30 \mathrm{~ns}$ of MD simulation. MSD data shows oscillatory behavior that was linearly fit for diffusion coefficient determination.

Table 3. Diffusion Coefficients for Kr/Xe through DD3R.

\begin{tabular}{ccc}
\hline Simulation & DD3R Cages & Diffusion Coefficient \\
\hline Pure Kr & Empty & $1.932 \times 10^{-13} \mathrm{~m}^{2} \mathrm{~s}^{-1}$ \\
$\mathrm{Kr}$ in Kr-Xe Mixture & Empty & $1.481 \times 10^{-13} \mathrm{~m}^{2} \mathrm{~s}^{-1}$ \\
$\mathrm{Kr}$ in Kr-Xe Mixture & Xe Pre-filled & $2.450 \times 10^{-13} \mathrm{~m}^{2} \mathrm{~s}^{-1}$ \\
Pure Xe & Empty & $6.809 \times 10^{-14} \mathrm{~m}^{2} \mathrm{~s}^{-1}$ \\
Xe in Kr-Xe Mixture & Empty & $5.674 \times 10^{-14} \mathrm{~m}^{2} \mathrm{~s}^{-1}$ \\
Xe in Kr-Xe Mixture & Xe Pre-filled & $4.567 \times 10^{-14} \mathrm{~m}^{2} \mathrm{~s}^{-1}$ \\
\hline
\end{tabular}

Diffusion coefficients, along the $\mathrm{x}$-direction, calculated from Figure 15 for pure $\mathrm{Kr}$ and for $\mathrm{Kr}$ in $\mathrm{Kr}-$ Xe mixture were $1.932 \times 10^{-13} \mathrm{~m}^{2} \mathrm{~s}^{-1}$ and $1.481 \times 10^{-13} \mathrm{~m}^{2} \mathrm{~s}^{-1}$. Due to the stronger interaction of Xe-zeolite leading to Xe blockage of the surface, the Kr diffusivity is lower in the mixture case. This is expected to change when Xe atoms advance through the membrane, instead of accumulating on the membrane surface, as the strong $\mathrm{Xe}-\mathrm{Kr}$ interaction would favor Xe to stay inside the membrane cages and allow $\mathrm{Kr}$ to permeate more easily. To study this assumption, DD3R inner cages were filled according to the manual filling scheme explained in the mixture effect on gas permeation previously. MSD results for this case, shown in Figure 15 in turquoise and olive for $\mathrm{Kr}$ and $\mathrm{Xe}$, respectively, show higher diffusion coefficient for $\mathrm{Kr}$ with $2.450 \times 10^{-13} \mathrm{~m}^{2} \mathrm{~s}^{-1}$ and lower diffusion for $\mathrm{Xe}$ at $4.567 \times 10^{-14} \mathrm{~m}^{2} \mathrm{~s}^{-1}$ in comparison to the pure gas simulations. This validates both the experimental trends and our molecular-level separation mechanism: Kr gas permeation through DD3R membrane is higher in the $\mathrm{Kr}$ / Xe gas mixture case compared to pure gases, because the attractive $\mathrm{Kr}-\mathrm{Xe}$ forces facilitates higher $\mathrm{Kr}$ permeationrates. 


\section{Discussion}

In runs (1) and (2), increasing pressure reduces the fraction of completely permeated $\mathrm{Kr}$, as shown in the inset plot of Figure 4. This agrees with the experimental trends in DD3R published by van den Bergh et al. [6], where Figure 4 of this reference shows the flux of $\mathrm{Kr}$ at $303 \mathrm{~K}$ increases with increasing pure $\mathrm{Kr}$ feed pressure between 100 and $400 \mathrm{kPa}$, i.e., between 1 and $4 \mathrm{~atm}$, but if normalized to the total number of atoms, there is hardly any change with feed pressure, actually a very slight decrease, while ours is $1.38 \%$ at 75 atm in run (1) going down to $1.16 \%$ at $150 \mathrm{~atm}$ in run (2), which agrees with the experimental trend. Our simulation results were carried out at much greater pressures, and the finite capacity of cages present in the MD simulation box, where cages were filled with maximum occupancy of $4 \mathrm{Kr}$ atom per DD3R alpha cage at both pressures are shown in Figure 5. Note that the fraction of $\mathrm{Kr}$ adsorbed went down from 25.05 to $16.50 \%$ upon increasing pressure. This is also the case for Xe in runs (4) and (5), where despite the absence of completely permeated Xe in these runs, Xe adsorbed on the membrane surface has gone down from 9.72 to $5.18 \%$ with increasing pressure in runs (4) and (5). This is also shown in Figure 8 , where Xe surface adsorption peaks are close to each other, signaling the higher adsorption at lower pressures. All these observations in our simulations are consistent with the experimental $\mathrm{Kr}$ data in DD3R [6] and the $\mathrm{Kr}$ and Xe data [3].

The effect of temperature on gas permeation was studied by increasing temperature to $425 \mathrm{~K}$ while keeping pressure at $150 \mathrm{~atm}$ by changing the number of atoms considered for the gas phase. Kr complete permeation has increased three-fold, shown clearly in the inset plot of Figure 6. The amount of $\mathrm{Kr}$ adsorbed in the membrane is lower at high temperatures as shown in Figure 7, where lower Kr occupancy numbers are observed, caused by higher kinetic energies that permit $\mathrm{Kr}$ to go over the energy barriers; $\mathrm{Kr}$ atoms more easily hop from one cage into another. Numerical values for runs (2) and (3) in Table 2 show this when examining the number of $\mathrm{Kr}$ atoms, which went down from 396 to $229 \mathrm{Kr}$ atoms when temperature was increased. This is similar for pure Xe where atom count on the adsorption surface decreased from $166 \mathrm{Xe}$ atoms in run (4) to 113 in run (6), shown also in Figure 10, where the density profile for Xe at high temperature is much lower than that at low temperature. The trend observed in DD3R in Figure 2 of [6] found the single component flux of $\mathrm{Kr}$ through DD3R decreases with increasing temperature between 200 and $300 \mathrm{~K}$, but does not change much between 300 and $400 \mathrm{~K}$, even very slightly increasing with temperature in this region, like our data $1.16 \%$ at $300 \mathrm{~K}$ in run (2) going to $3.69 \%$ at $425 \mathrm{~K}$ in run (3). Our MD results do not carry Xe all the way to complete permeation, but when we look inside the membrane region in Table 2, simulations (4) and (6), we find 5.18\% at $300 \mathrm{~K}$ going up to $7.62 \%$ at $425 \mathrm{~K}$, which is the same trend as seen in DD3R [3].

After reviewing the effects of pressure and temperature on permeation of pure gases, we moved into the equimolar gas permeation, which is the major focus of this study. According to experimental results, $\mathrm{Kr}$ complete permeation increases in going from pure to mixture gas conditions [3]. Following our conventional simulation setup, starting MD simulations with empty membrane cages, our results from run (7) did not exhibit the same experimental trends with fraction of completely permeated $\mathrm{Kr}$ decreasing from $1.16 \%$ in the pure gas (2) to $0.88 \%$ in the gas mixture as shown by the dark blue dashed line in Figure 12. As discussed earlier, this is caused by the slow diffusion process of $\mathrm{Xe}$, which results in its accumulation on the membrane surface rather than complete permeation. The blockage stops $\mathrm{Kr}$ diffusion, as shown in Figure 14a. This artefactual result is attributed to the fact the system was not simulated at equilibrium adsorption conditions. Xe prefers to occupy zeolite cages as shown by experimental adsorption isotherms in the literature, so to carry out permeation simulations under steady state conditions, the Xe occupancies of the cages should be what would have been in steady state, before the start of the permeation simulation.

To circumvent this, we followed the manual Xe pre-filling scheme explained previously to explore the effect of $\mathrm{Xe}-\mathrm{Kr}$ attractive forces on $\mathrm{Kr}$ permeation in the gas mixture. While the idea of simulating adsorption equilibrium conditions for the membrane structure is of 
actual relevance to real-life experiments, the manual Xe pre-filling proposed in this study and used in run (8) was successful in reproducing experimental trends for $\mathrm{Kr}$ permeation; ref. [3] that is, the fraction of completely permeated $\mathrm{Kr}$ has doubled compared to the pure simulation in run (2). On the other hand, Xe kept the same behavior as in the conventional simulation method with no complete permeation as shown in Figure 12, where pre-filled Xe is showing a uniform distribution in the inner cages of the membrane. These results provide a clear picture of the mechanism of the observed separation. Xe because of its stronger affinity for the zeolite occupies the cavities of the cages. The Xe in the cages then because of their stronger interaction with $\mathrm{Kr}$, promote their transport through the zeolite membrane. Thus, $\mathrm{Kr}$ permeates at a higher rate when Xenon is present than when it is not. This is often not the case because the larger molecules can block the zeolite surface and thus prevent the smaller molecules from entering the membrane.

In addition, the value $1.932 \times 10^{-13} \mathrm{~m}^{2} \mathrm{~s}^{-1}$ at $300 \mathrm{~K}$ for $\mathrm{Kr}$ in DD3R reported here in Table 3 from the MSD data is close to the experimental value of $(3.1 \pm 1.3) \times 10^{-13} \mathrm{~m}^{2} \mathrm{~s}^{-1}$ at $300 \mathrm{~K}$ for $\mathrm{Kr}$ in DD3R from reference [6] and also close to the value calculated using Maxwell Stefan model combined with new thermodynamic factors, and different models for adsorption isotherms in reference [23] $(2.03-2.09) \times 10^{-13} \mathrm{~m}^{2} \mathrm{~s}^{-1}$ at $300 \mathrm{~K} \mathrm{for} \mathrm{Kr}$ in DD3R. This is a further validation of the simulations in this work.

\section{Conclusions}

The separation of $\mathrm{Kr} / \mathrm{Xe}$ radioactive waste is a process of enormous benefit for environmental and economic reasons. Using zeolites for such separation has been showing promising results, with some zeolites showing separation factors as high as $51 \mathrm{for} \mathrm{Kr} / \mathrm{Xe}$ separations [2]. This work examined DD3R zeolite for such a separation process. This computational study has confirmed almost all trends found in experimental work discussed here $\mathrm{k}$. While this study only reproduces trends for experimental work, the next stage of this project will be exploring the equilibrium adsorption conditions in the membrane from the start of MD simulation in addition to using Ar as sweep gas to promote $\mathrm{Kr} / \mathrm{Xe}$ permeation to study the steady-state conditions to obtain separation factors similar to those observed in experimental work.

Author Contributions: Conceptualization, S.M.; Methodology, B.J.B.; Software, B.J.B.; Validation, X.W., C.J.J. and S.M.; Formal analysis, B.J.B.; Investigation, B.J.B. and C.J.J.; Resources, S.M.; Data curation, B.J.B.; Writing—original draft preparation, B.J.B.; Writing-review and editing, B.J.B., X.W., C.J.J. and S.M.; Visualization, B.J.B.; Supervision, S.M. All authors have read and agreed to the published version of the manuscript.

Funding: B.J.B. is supported by King Abdulaziz University, Jeddah, Saudi Arabia.

Institutional Review Board Statement: Not applicable.

Informed Consent Statement: Not applicable.

Data Availability Statement: The data that support the findings of this study are available from the corresponding author upon reasonable request.

Conflicts of Interest: The authors declare no conflict of interest.

\section{References}

1. Jameson, C.J.; Jameson, A.K.; Lim, H.-M. Competitive Adsorption of Xenon and Krypton in Zeolite NaA: 129Xe Nuclear Magnetic Resonance Studies and Grand Canonical Monte Carlo Simulations. J. Chem. Phys. 1997, 107, 4364-4372. [CrossRef]

2. Wang, X.; Zhou, T.; Zhang, P.; Yan, W.; Li, Y.; Peng, L.; Veerman, D.; Shi, M.; Gu, X.; Kapteijn, F. High-Silica CHA Zeolite Membrane with Ultra-High Selectivity and Irradiation Stability for Krypton/Xenon Separation. Angew. Chem. Int. Ed. 2021, 60, 9032-9037. [CrossRef]

3. Wang, X.; (Nanjing Tech University, Nanjing, China). Personal communication, 2020.

4. Wang, X.; Zhang, Y.; Wang, X.; Andres-Garcia, E.; Du, P.; Giordano, L.; Wang, L.; Hong, Z.; Gu, X.; Murad, S.; et al. Xenon Recovery by DD3R Zeolite Membranes: Application in Anaesthetics. Angew. Chem. Int. Ed. 2019, 58, 15518-15525. [CrossRef] [PubMed] 
5. van de Graaf, J.M.; Kapteijn, F.; Moulijn, J.A. Methodological and Operational Aspects of Permeation Measurements on Silicalite-1 Membranes. J. Membr. Sci. 1998, 144, 87-104. [CrossRef]

6. van den Bergh, J.; Zhu, W.; Gascon, J.; Moulijn, J.A.; Kapteijn, F. Separation and Permeation Characteristics of a DD3R Zeolite Membrane. J. Membr. Sci. 2008, 316, 35-45. [CrossRef]

7. Martínez, L.; Andrade, R.; Birgin, E.G.; Martínez, J.M. PACKMOL: A package for building initial configurations for molecular dynamics simulations. J. Comput. Chem. 2009, 30, 2157-2164. [CrossRef] [PubMed]

8. Baerlocher, C.; McCusker, L.B. Database of Zeolite Structures. Available online: http://www.iza-structure.org/databases/ (accessed on 8 October 2021).

9. Murad, S.; Jia, W.; Krishnamurthy, M. Ion-Exchange of Monovalent and Bivalent Cations with NaA Zeolite Membranes: A Molecular Dynamics Study. Mol. Phys. 2004, 102, 2103-2112. [CrossRef]

10. Qu, F.; Shi, R.; Peng, L.; Zhang, Y.; Gu, X.; Wang, X.; Murad, S. Understanding the Effect of Zeolite Crystal Expansion/Contraction on Separation Performance of NaA Zeolite Membrane: A Combined Experimental and Molecular Simulation Study. J. Membr. Sci. 2017, 539, 14-23. [CrossRef]

11. Hinkle, K.R.; Wang, X.; Gu, X.; Jameson, C.J.; Murad, S. Computational Molecular Modeling of Transport Processes in Nanoporous Membranes. Processes 2018, 6, 124. [CrossRef]

12. Chen, C.; Cheng, Y.; Peng, L.; Zhang, C.; Wu, Z.; Gu, X.; Wang, X.; Murad, S. Fabrication and Stability Exploration of Hollow Fiber Mordenite Zeolite Membranes for Isopropanol/Water Mixture Separation. Microporous Mesoporous Mater. 2019, 274, 347-355. [CrossRef]

13. Thompson, A.P.; Aktulga, H.M.; Berger, R.; Bolintineanu, D.S.; Michael Brown, W.; Crozier, P.S.; in't Veld, P.J.; Kohlmeyer, A.; Moore, S.G.; Nguyen, T.D.; et al. LAMMPS-A Flexible Simulation Tool for Particle-Based Materials Modeling at the Atomic, Meso, and Continuum Scales. Comput. Phys. Commun. 2021, 271, 108171. [CrossRef]

14. Momma, K.; Izumi, F. VESTA: A Three-Dimensional Visualization System for Electronic and Structural Analysis. J. Appl. Crystallogr. 2008, 41, 653-658. [CrossRef]

15. Humphrey, W.; Dalke, A.; Schulten, K. VMD: Visual Molecular Dynamics. J. Mol. Graph. 1996, 14, 33-38. [CrossRef]

16. Parkes, M.V.; Demir, H.; Teich-McGoldrick, S.L.; Sholl, D.S.; Greathouse, J.A.; Allendorf, M.D. Molecular Dynamics Simulation of Framework Flexibility Effects on Noble Gas Diffusion in HKUST-1 and ZIF-8. Microporous Mesoporous Mater. 2014, 194, 190-199. [CrossRef]

17. Kitani, S.; Takada, J. Adsorption of Krypton and Xenon on Various Adsorbents. J. Nucl. Sci. Technol. 1965, 2, 51-56. [CrossRef]

18. Guillot, B.; Sator, N. Noble Gases in High-Pressure Silicate Liquids: A Computer Simulation Study. Geochim. Cosmochim. Acta 2012, 80, 51-69. [CrossRef]

19. Fatriansyah, J.F.; Dhaneswara, D.; Kuskendrianto, F.R.; Abdurrahman, M.H.; Yusuf, M.B.; Abdillah, F.A. The Effect of Temperature and Pressure on Nitrogen Adsorption in Amorphous Silica. J. Phys. Conf. Ser. 2021, 2070, 012045. [CrossRef]

20. Tomita, T.; Nakayama, K.; Sakai, H. Gas Separation Characteristics of DDR Type Zeolite Membrane. Microporous Mesoporous Mater. 2004, 68, 71-75. [CrossRef]

21. Auerbach, S.M.; Carrado, K.A.; Dutta, P.K. Handbook of Zeolite Science and Technology; CRC Press: Boca Raton, FL, USA, 2003; ISBN 978-0-203-91116-7.

22. Shang, J.; Li, G.; Singh, R.; Xiao, P.; Liu, J.Z.; Webley, P.A. Determination of Composition Range for "Molecular Trapdoor" Effect in Chabazite Zeolite. J. Phys. Chem. C 2013, 117, 12841-12847. [CrossRef]

23. Lito, P.F.; Santiago, A.S.; Cardoso, S.P.; Figueiredo, B.R.; Silva, C.M. New Expressions for Single and Binary Permeation through Zeolite Membranes for Different Isotherm Models. J. Membr. Sci. 2011, 367, 21-32. [CrossRef] 